Artículo Original/ Original Article

http://dx.doi.org/10.18004/mem.iics/1812-9528/2020.018.02.63

\title{
I ntervención Educativa-Nutricional sobre hábitos alimentarios aplicada a escolares de Asunción, Paraguay
}

\author{
* María Villagra ${ }^{1}$, Eliana Meza ${ }^{2}$, Dora Villalba ${ }^{3}$ \\ ${ }^{1}$ Universidad Nacional de Asunción, Facultad de Ciencias Veterinarias, Departamento de Pesca y \\ Acuicultura. Paraguay \\ ${ }^{2}$ Universidad Nacional de Asunción, Centro Multidisciplinario de Investigaciones Tecnológicas (CEMIT), \\ Departamento de Biotecnología. Paraguay \\ ${ }^{3}$ Universidad Nacional de Asunción, Rectorado. Paraguay
}

Cómo referenciar este artículo/ How to reference this article:
Villagra M, Meza E, Villalba D. Intervención Educativa-Nutricional sobre hábitos alimentarios aplicada a escolares de Asunción, Paraguay. Mem. Inst. Investig. Cienc. Salud. 2020; 18(2): 63-73

\section{RE S U M E N}

Determinados hábitos alimentarios y estilos de vida adquiridos en la infancia se asocian al riesgo de padecer en la edad adulta enfermedades crónicas no transmisibles. En Paraguay, actualmente, el estado nutricional escolar representa un problema de salud pública. Es importante establecer la implementación de intervenciones educativas-nutricionales, enfocadas a la mejoría de la calidad de hábitos alimentarios, como posible estrategia para la prevención de estas enfermedades. Para evaluar cambios en la calidad de hábitos alimentarios en niños/as de 10-13 años, se llevó a cabo una intervención educativa-nutricional en la Escuela República del Brasil, Asunción-Paraguay. Este ensayo comunitario incluyó a 42 escolares y sus padres. Al inicio y final de la intervención, se estimó el peso, talla, estado nutricional, calidad de hábitos alimentarios y tipo de actividad física en estos escolares. Durante el ensayo que duró de marzo a abril del 2019, a los niños se les impartió una charla semanal de 60 minutos mientras que los padres tuvieron dos charlas, al principio y final del ensayo, donde describieron su calidad de hábitos alimentarios. Finalizada la intervención, se observó que hubo mejoras significativas en la calidad de hábitos alimentarios. Aquellos niños que inicialmente contaban con necesidad de mejorar hábitos posteriormente pasaron a una óptima calidad de hábitos alimentarios, y el mismo resultado se observó en sus padres. En conclusión, la intervención generó cambios en la calidad de hábitos alimentarios de niños, padres y madres, pudiendo establecerla como parte del programa nutricional escolar, para generar consciencia sobre los hábitos saludables y prevención de enfermedades.

Palabras clave: calidad de hábitos alimentarios, actividad física, niños escolares, estado nutricional, intervención.

\section{Educational-Nutritional I ntervention on eating habits applied to school children of Asuncion, Paraguay}

\section{A B S T R A C T}

Certain eating habits and lifestyles acquired in childhood are associated with the risk of suffering chronic non-communicable diseases in adulthood. Currently in Paraguay, school nutritional status represents a public health problem. It is important to

Los autores expresan que no hay conflictos de intereses al redactar el presente manuscrito.

Fuente de financiación propia.

Fecha de recepción: junio 2020. Fecha de aceptación: julio 2020

* Autor correspondiente: María Villagra. Universidad Nacional de Asunción Facultad de Ciencias Veterinarias, *Departamento de Pesca y Acuicultura. Paraguay. Ruta Mcal. Estigarribia km 11. +595971270740 .

Email: masterv26@gmail.com 
establish the implementation of educational-nutritional interventions focused on improving the quality of eating habits, as a possible strategy for the prevention of those diseases. In order to evaluate changes in the quality of eating habits in children who were 10-13 years old, a nutritional educational intervention was carried out in the República del Brasil School of Asunción, Paraguay. This community study included 42 children and their parents. At the beginning and end of the intervention the weight, size, nutritional status, quality of eating habits and type of physical activity were estimated in these school children. During the study, that lasted from March to April 2019, the children were given weekly talks of 60 minutes, while the parents had two talks at the beginning and end of the intervention in which they described their quality of eating habits. After the intervention, it was observed that there were significant improvements in the quality of eating habits. Those children who initially had the need to improve habits subsequently changed to an optimal quality of eating habits, and the same result was observed in their parents. In conclusion, the intervention generated changes in the quality of eating habits of these children and their parents and it was established as part of the school nutritional program in order to create awareness about healthy habits and prevention of diseases.

Keywords: quality of eating habits, physical activity, school children, nutritional status, intervention.

\section{NTRODUCCIÓN}

Determinados hábitos alimentarios y estilos de vida propician la tendencia a la obesidad de la población escolar, con la consiguiente predisposición a padecer en la edad adulta de enfermedades crónicas no transmisibles (ECNT) como: hipertensión arterial, diabetes mellitus, cáncer, obesidad, entre otros ${ }^{(1)}$. El sobrepeso y la obesidad son considerados mundialmente como factores de riesgo, produciendo millones de muertes al año ${ }^{(2)}$, así como la desnutrición de niños en países en vías de desarrollo, es la causante de 3,1 a 5 millones de mortalidad infantil, representando un $45 \%$ de muertes a nivel mundial ${ }^{(3)}$, en Paraguay, la prevalencia de obesidad ha aumentado progresivamente durante los últimos años, siendo los hábitos alimentarios y estilos de vida sedentarios unos de los factores de riesgo ${ }^{(4)}$.

Actualmente, a nivel país, se ha establecido el Plan Nacional de Acción para la Prevención y Control de Enfermedades Crónicas No Transmisibles 2014-2024, que contempla estrategias para la prevención y el monitoreo de estas enfermedades ${ }^{(5)}$; igualmente, se han elaborado Guías Alimentarias, que contienen recomendaciones orientadas a promover dietas saludables, estilos de vida sanos y prevenir las enfermedades crónicas de la población ${ }^{(6)}$; así mismo, se ha implementado el Programa de Alimentación Escolar, que busca lograr una alimentación nutricionalmente adecuada, una educación alimentaria y nutricional que favorezcan el desarrollo de estilos de vida saludable en escolares ${ }^{(7)}$. De manera a tratar de prevenir situaciones que eleven el riesgo de mortalidad, en la actualidad, a nivel internacional se realizan las intervenciones escolares, que constituyen una de las estrategias más importantes para mejorar la calidad de los hábitos alimentarios que determinan el estado nutricional en la población escolar actual, es fundamental idear o reforzar programas de educación nutricional que puedan desarrollarse en diferentes ámbitos y que uno de los principales elementos del aprendizaje sea la motivación y que el mismo se utilice como herramienta educativa para enseñar habilidades sociales de forma que sea más fácil conseguir que las actitudes se transformen en conductas ${ }^{(8)}$. Un estudio en el cual se realizó una intervención educativa sobre un grupo de 54 alumnos de un Centro de Educación de Sevilla - España, por parte de un profesional sanitario, en el cual se estimaron mediante una encuesta el peso, talla, adherencia a la Dieta Mediterránea, grado de actividad física, conocimientos en alimentación y hábitos de vida saludables, y luego de dos meses se reevaluaron los conocimientos de alimentación y hábitos de vida saludable en todos los alumnos; arrojó como resultado un aumento estadísticamente significativo en conocimientos relativos a alimentación y hábitos de vida saludable, hacen mención a que las actividades educativas de apoyo dentro del programa escolar de estilos de vida saludable por parte de profesionales 
sanitarios pueden suponer un beneficio adicional en las estrategias encaminadas a mejorar el estado nutricional del escolar en el medio ${ }^{(8)}$.

Teniendo en cuenta los antecedentes tanto a nivel país como a nivel internacional, destacando la situación actual del estado nutricional en la población de niños escolares en Paraguay, siendo éste un problema de salud pública y considerando la necesidad de contar con programas nutricionales educativos enfocados a la mejoría de la calidad de hábitos alimentarios, motiva la realización de esta investigación de carácter experimental que plantea la educación nutricional como punto de partida para inculcar los hábitos alimentarios y estilos de vida saludables, en el cual los escolares sean capaces de promover actitudes positivas hacia los hábitos saludables, presentando comportamientos duraderos y así mejorar los niveles de salud ${ }^{(9,10)}$, se trata de no centrar toda la atención solo en el peso corporal, sino crear un impacto positivo en cuanto a la salud en general, vivir activamente, tener una alimentación normal y balanceada, crear un ambiente nutritivo que ayude al niño a reconocer y obtener su propio entorno saludable sostenidos en el tiempo ${ }^{(11,12)}$. El objetivo de este trabajo fue describir los cambios en la calidad de hábitos alimentarios en niños/as escolares de entre 10 a 13 años tras la aplicación de una intervención educativa nutricional, a través de una serie de charlas, con una duración de 8 semanas, divididas en sesiones semanales de 60 minutos, se trataron temas relacionados a la salud: alimentación, relajación y actividades físicas.

\section{MATERI ALES Y MÉTODOS}

Diseño: Ensayo comunitario con 8 semanas de duración (marzo a abril del 2019), en el cual se realizaron las siguientes mediciones:

- Antropométricas en niños: toma de medidas de peso y talla

- Calidad de Hábitos Alimentarios para niños y padres: aplicación del Test de KIDMED $^{(13)}$

- Actividad Física en niños: aplicación del Cuestionario sobre Actividad Física ${ }^{(14)}$.

Muestra: 42 niños/as de 10 a 13 años del $6^{\circ}$ Grado Sección "A" y "B", de la Escuela Básica $\mathrm{N}^{\circ} 3$ República del Brasil, Asunción - Paraguay y sus respectivos padres. Se optó por aplicar esta intervención al grupo de niños/as con la edad mencionada, teniendo en cuenta un factor de suma relevancia para las escuelas públicas como lo es el factor del "tiempo disponible", con el cual no contaban los demás grados pertenecientes a la institución.

\section{Aspectos éticos}

Todas las actividades realizadas fueron aprobadas por la Comisión de Ética de la Facultad de Ciencias Químicas de la Universidad Nacional de Asunción. Código CEI: 236/16, y autorizado por los Directivos de la Escuela Básica Nº 3 República del Brasil, previo consentimiento de los padres de los niños.

\section{Fases del Estudio}

$1^{\circ}$ Fase: Diagnóstico de situación: Charla informativa a los padres (sobre el método y la importancia y autorización del trabajo con los niños) y aplicación del Test de KIDMED ${ }^{(13)}$ (para conocer la calidad de sus hábitos alimentarios). En cuanto a los niños, se procedió a tomar medidas de (Peso y Talla), aplicación del Test de KIDMED ${ }^{13}$ y Cuestionario sobre Actividad Física ${ }^{(14)}$.

$2^{\circ}$ Fase: Intervención: Inicio de Sesión de Charlas semanales ( 1 charla/semana), 60 minutos de duración, durante un periodo de 8 semanas. Antes de iniciar cada charla se presentaba el tema de la misma y para facilitar la recepción, captar la atención de los niños y que sea una actividad de aprendizaje y diversión, se realizaba un juego didáctico previo (Ej.: sopa de letras sobre el tema de la sesión). Los temas de las charlas: Guías Alimentarias; Clasificación de alimentos; Frutas, un mundo de colores y formas; Verduras; Buenos días, ¿Desayunamos?; Hola, qué te parece si empezamos a movernos?; Importancia del Agua, Menos gaseosas, más jugos naturales, por qué?.

Transcurridas las 8 semanas, y en la $8^{\circ}$ Sesión, tras exponer el último tema, se realizaron nuevamente las tomas de medidas (Peso y Talla), aplicación del Test de 
KIDMED $^{(13)}$ y Cuestionario sobre Actividad Física ${ }^{(14)}$, a los efectos de verificar si hubo cambios en algunos de los indicadores medidos anteriormente.

$3^{\circ}$ Fase: Presentación de resultados: Se realizó la $2^{\circ}$ Charla con los Padres, se presentaron los resultados obtenidos con los niños y se realizó nuevamente el Test de KIDMED $^{(13)}$ a padres.

\section{Variables e Instrumentos}

Registro de variables: se utilizaron planillas elaboradas por la investigadora, en la cual se especificaron los datos personales, socio-demográficos y antropométricos.

Datos Antropométricos para niños: Peso, se obtuvo a partir de la utilización de una Balanza Omron, con capacidad de peso hasta $150 \mathrm{~kg}$ y graduación de $0.1 \mathrm{~kg}$, en cual el niño se encontraba con el mínimo de ropa, cuidando una posición simétrica y central en la plataforma de la báscula; igualmente, para la Talla, se utilizó un tallímetro de Nutrisys, de 2 metros, cuidando de que el niño se encuentre de pie, sin zapatos y sin adornos en la cabeza, con los talones unidos a los ejes longitudinales de ambos pies. El niño mantuvo la cabeza de manera que el plano de Frankfort se conserve horizontal, se realizó la lectura aproximándose a milímetros, aceptando una variación de $1 \mathrm{~mm}$. La valoración del estado nutricional se realizó a partir del cálculo del IMC/EDAD, en la que se utilizaron las Curvas de Crecimiento para niños/as de 519 años, OMS - 2007(15), así mismo, para la evaluación de TALLA/EDAD, se utilizaron las Curvas de Crecimiento para niños/as de 5-19 años, OMS - 2007(15).

Calidad de Hábitos Alimentarios para niños y padres: se utilizó el Test de KIDMED ${ }^{(13)}$, que constó de 16 preguntas, con sus respectivos puntajes a cada pregunta, que clasifica en tres niveles de calidad sobre hábitos alimentar\&os,

puntos para una Dieta de Óptima Calidad, 4 - 7 puntos una Necesidad de Mejorar el Patrón Alimentario y $\leq 3$ puntos una Dieta de Muy Baja Calidad. Seguidamente, para los Padres se utilizó el mismo Test de KIDMED ${ }^{(13)}$.

Tipo de Actividad Física para niños: se utilizó un Cuestionario de Actividad Física ${ }^{(14)}$, que califica como Actividad Física Buena de 7 - 10 puntos, Actividad Física Regular de $4-6$ puntos y Actividad Física Baja $<3$ puntos.

\section{Análisis de los datos}

Los datos fueron digitalizados, procesados y analizados con Microsoft Office ${ }^{\circledR}$ y Excel ${ }^{\circledR} 2007$ (EE.UU.). Tras comprobar la consistencia de la base de datos, se procedió al análisis de los mismos. Los datos cuantitativos con distribución normal se resumieron en: promedio y desviaciones estándar (DE) y aquellos con distribución no normal como mediana (Me), mínimo y máximo. Los datos cualitativos, se resumieron en frecuencia $(n)$ porcentaje (\%). Para la comparación de medias y/o varianzas se empleó la prueba "t-Student" para muestras apareadas o la suma de Wilcoxon para muestras apareadas; para comparar los datos cualitativos se utilizó la prueba de "ChiCuadrado".

\section{RESULTADOS}

En la Tabla 1 se describe el estado nutricional categorizado de los niños. Se observaron disminuciones en cuanto a aquellos escolares que contaban con obesidad, ya que inicialmente se encontraban diez niños (24\%) y al término de la intervención, sólo quedaron siete $(17 \%)$ con el referido estado. En cuanto a los escolares que contaban con sobrepeso, no arrojaron cambios, sin embargo, en lo que respecta al estado adecuado, si se encontraron diferencias al final de la intervención, ya que se muestra un aumento del porcentaje de escolares (66\%) respecto al valor inicial (55\%). Por otro lado, para el indicador de riesgo de desnutrición, se observó una disminución al término de las charlas $(5 \%)$, teniendo en cuenta que al principio incluían el doble de escolares ( $9 \%$ ) en dicha categoría. Para el indicador de talla/edad, no se encontraron cambios. En la Tabla 2 se observa la calidad de hábitos alimentarios de los escolares. Al comparar las medianas de los puntajes obtenidos antes y después de la intervención en los escolares, se observó que existen diferencias significativas entre los grupos ( $p<0,001$ prueba de Wilcoxon). Con respecto a la categoría óptima hubo un aumento de escolares al finalizar la misma, en cuanto a la variable necesidad 
Villagra et al Intervención Educativa-Nutricional sobre hábitos alimentarios aplicada a escolares...

de mejorar y no óptima se observó una disminución de niños que se encontraban con esa calidad de hábitos alimentarios.

Tabla 1: Estado Nutricional de niños/as de la Escuela Básica $N^{\circ} 3$ República del Brasil al inicio y al final de la Intervención

\begin{tabular}{lllll}
\hline \multirow{2}{*}{ Variable } & \multicolumn{2}{l}{ I nicial } & \multicolumn{2}{l}{ Final } \\
& N & \% & N & $\%$ \\
\hline I MC/ EDAD & & & & \\
Obesidad & 10 & 24 & 7 & 17 \\
Sobrepeso & 5 & 12 & 5 & 12 \\
Adecuado & 23 & 55 & 28 & 66 \\
Riesgo de Desnutrición & 4 & 9 & 2 & 5 \\
Desnutrición & - & - & - & - \\
\hline Total & 42 & 100 & 42 & 100 \\
\hline Talla/ Edad & & & & \\
Talla adecuada & 38 & 91 & 38 & 91 \\
Riesgo de talla baja & 4 & 9 & 4 & 9 \\
Talla baja & - & - & - & - \\
Talla muy baja & - & - & - & - \\
\hline Total & 42 & 100 & 42 & 100 \\
\hline
\end{tabular}

Tabla 2: Calidad de hábitos alimentarios de niños/as de la Escuela Básica $\mathrm{N}^{\circ} 3$ República del Brasil al inicio y al final de la intervención $* p<0,001$ (prueba de Wilcoxon).

\begin{tabular}{|c|c|c|c|c|c|c|c|}
\hline \multicolumn{2}{|c|}{ Variable } & \multicolumn{3}{|c|}{ Inicial } & \multicolumn{3}{|c|}{ Final } \\
\hline $\begin{array}{l}\text { Calidad de } \\
\text { hábitos } \\
\text { alimentarios }\end{array}$ & Rango & $\mathbf{N}$ & $\begin{array}{c}\text { Promedio de } \\
\text { puntaje obtenido } \\
( \pm \mathrm{DE})\end{array}$ & $\%$ & $\mathbf{N}$ & $\begin{array}{c}\text { Promedio de } \\
\text { puntaje obtenido } \\
( \pm D E)\end{array}$ & $\%$ \\
\hline Optima & $\geq 8$ & 16 & $\begin{array}{l}10,25 \\
( \pm 1,53)\end{array}$ & 38 & 34 & $\begin{array}{l}9,7 \\
( \pm 1,63)\end{array}$ & 81 \\
\hline $\begin{array}{l}\text { Necesidad } \\
\text { de mejorar }\end{array}$ & 4 a 7 & 21 & $\begin{array}{l}5,76 \\
( \pm 1,00)\end{array}$ & 50 & 8 & $\begin{array}{l}6,5 \\
( \pm 0,92)\end{array}$ & 19 \\
\hline No Optima & $\leq 3$ & 5 & $\begin{array}{l}2,80 \\
( \pm 0,45)\end{array}$ & 12 & - & - & - \\
\hline Total & & 42 & & 100 & 42 & & 100 \\
\hline
\end{tabular}

Al comparar las medianas de los puntajes obtenidos antes y después de la intervención en los escolares, se observó que existen diferencias significativas entre los grupos. ( $p<0,001$ prueba de Wilcoxon).

Seguidamente, en la Tabla 3 se describen las actividades físicas categorizadas de los escolares. Se puede observar que existe, al final de la intervención, una disminución en cuanto a la categoría de actividad física regular y un aumento en la actividad física buena, comparándolo con los resultados iniciales. Por otro lado, en la Tabla 4 se muestran los resultados arrojados en cuanto a la relación entre Estado Nutricional y Actividad Física de escolares tras la intervención. Al analizar si existe asociación entre el estado nutricional de los escolares post intervención y el tipo de actividad física de los mismos tras la intervención, no se encontró relación entre estas variables $(p=0,465$ Chi cuadrado). Igualmente, se observa que ciertos escolares presentan obesidad con actividad física buena y regular, así mismo, algunos niños que tenían sobrepeso arrojaron también actividad física buena y regular, en cambio la mayor parte de aquellos escolares que contaban con estado nutricional adecuado 
Villagra et al Intervención Educativa-Nutricional sobre hábitos alimentarios aplicada a escolares...

presentaban actividad física regular y buena, por otro lado, se constató una menor cantidad de niños con riesgo de desnutrición que presentaron una buena actividad física. No hubo evidencias de niños que presentaron desnutrición o baja actividad física.

Tabla 3: Actividades Físicas de niños/as al inicio y al final de la intervención.

\begin{tabular}{lllll}
\hline $\begin{array}{l}\text { Variable } \\
\text { Actividad Física }\end{array}$ & N & $\begin{array}{c}\text { I nicial } \\
\%\end{array}$ & N & $\begin{array}{c}\text { Final } \\
\%\end{array}$ \\
\hline Baja & - & - & - & - \\
Regular & 40 & 95 & 19 & 45 \\
Buena & 2 & 5 & 23 & 55 \\
Total & 42 & 100 & 42 & 100 \\
\hline
\end{tabular}

Tabla 4: Relación entre Estado Nutricional y Actividad Física de escolares tras la intervención * $p=0,465$ Chi cuadrado.

\begin{tabular}{llllll}
\hline \multirow{2}{*}{$\begin{array}{l}\text { Estado nutricional } \\
\text { de escolares }\end{array}$} & & \multicolumn{3}{l}{ Tipo de Actividad Física } & \multirow{2}{*}{ Total } \\
\cline { 2 - 5 } & & Bajo & Regular & Buena & \\
\hline Obesidad & $\mathrm{N}$ & 0 & 4 & 3 & 7 \\
& $\%$ & 0 & 57,1 & 42,9 & 16,7 \\
\hline Sobrepeso & $\mathrm{N}$ & 0 & 3 & 2 & 5 \\
& $\%$ & 0 & 60,0 & 40,0 & 12,0 \\
\hline Adecuado & $\mathrm{N}$ & 0 & 12 & 16 & 28 \\
& $\%$ & 0 & 42,9 & 57,7 & 66,7 \\
\hline Riesgo de & $\mathrm{N}$ & 0 & 0 & 2 & 2 \\
desnutrición & $\%$ & 0 & 0 & 100 & 5,0 \\
\hline Desnutrición & $\mathrm{N}$ & 0 & 0 & 0 & 0 \\
& $\%$ & 0 & 0 & 0 & 0 \\
\hline Total & $\mathrm{N}$ & 0 & 19 & 23 & 42 \\
& $\%$ & 100 & 100 & 100 & 100 \\
\hline
\end{tabular}

Al analizar si existe asociación entre el estado nutricional de los escolares post intervención y el tipo de actividad física de los mismos tras la intervención, no se encontró relación entre estas variables ( $p=0,465$ Chi cuadrado).

En la Tabla 5 se presenta la calidad de hábitos alimentarios de madres de niños/as de la Escuela Básica $N^{\circ} 3$ República del Brasil al inicio y al final de la intervención. Al comparar las medianas de los puntajes obtenidos antes y después de la intervención en las madres, observamos que existen diferencias significativas entre los grupos $(p<0,001$ prueba de Wilcoxon). Los resultados arrojados demuestran que existe un aumento en lo que respecta a las madres que contaban con una óptima calidad de hábitos alimentarios al finalizar la intervención, así mismo, se observa una disminución en la cantidad de personas con necesidad de mejorar los hábitos y calidad de hábitos no óptima. Finalmente, teniendo en cuenta la calidad de hábitos alimentarios de padres de niños/as de la Escuela Básica $N^{\circ} 3$ República del Brasil al inicio y al final de la intervención, correspondiente a la Tabla 6, al comparar las medianas de los puntajes obtenidos antes y después de la intervención en los padres, observamos que existen diferencias significativas entre los grupos $(p<0,001$ prueba de Wilcoxon). Los resultados arrojaron una elevada cantidad de padres con una óptima calidad de hábitos alimentarios al final del estudio, igualmente, se presentó una disminución en la cantidad de personas que tenían necesidad de mejorar los hábitos así como para aquellos que contaban con una calidad de hábitos que no era óptima. 
Villagra et al Intervención Educativa-Nutricional sobre hábitos alimentarios aplicada a escolares...

Tabla 5: Calidad de hábitos alimentarios de madres de niños/as de la Escuela Básica $N^{\circ} 3$ República del Brasil al inicio y al final de la intervención* $p<0,001$ (prueba de Wilcoxon)

\begin{tabular}{|c|c|c|c|c|c|c|c|}
\hline $\begin{array}{l}\text { Variable } \\
\text { Calidad de } \\
\text { hábitos } \\
\text { alimentarios }\end{array}$ & Rango & $\mathbf{N}$ & $\begin{array}{l}\text { Inicial } \\
\text { Promedio } \\
\text { de puntaje } \\
\text { obtenido } \\
\text { ( } \pm \text { DE) }\end{array}$ & $\%$ & $\mathbf{N}$ & $\begin{array}{l}\text { Final } \\
\text { Promedio } \\
\text { de puntaje } \\
\text { obtenido } \\
\text { ( } \pm \text { DE) }\end{array}$ & $\%$ \\
\hline Optima & $\geq 8$ & 16 & $\begin{array}{l}10,56 \\
( \pm 1,15)\end{array}$ & 38 & 28 & $\begin{array}{l}10,1 \\
( \pm 1,64)\end{array}$ & 67 \\
\hline $\begin{array}{l}\text { Necesidad de } \\
\text { mejorar }\end{array}$ & 4 a 7 & 21 & $\begin{array}{l}6,19 \\
( \pm 1,12)\end{array}$ & 50 & 13 & $\begin{array}{l}6,3 \\
( \pm 1,1)\end{array}$ & 31 \\
\hline No Optima & $\leq 3$ & 5 & $\begin{array}{l}2,4 \\
( \pm 0,55)\end{array}$ & 12 & 1 & $\begin{array}{l}2 \\
( \pm 2)\end{array}$ & 2 \\
\hline Total & & 42 & & 100 & 42 & & 100 \\
\hline
\end{tabular}

Al comparar las medianas de los puntajes obtenidos antes y después de la intervención en las madres, observamos que existen diferencias significativas entre los grupos. ( $p<0,001$ prueba de Wilcoxon)

Tabla 6. Calidad de hábitos alimentarios de padres de niños/as de la Escuela Básica $N^{\circ} 3$ República del Brasil al inicio y al final de la intervención* $p<0,001$ (prueba de Wilcoxon).

\begin{tabular}{|c|c|c|c|c|c|c|c|}
\hline Variable & & & nal & & & & \\
\hline $\begin{array}{l}\text { Calidad de } \\
\text { hábitos } \\
\text { alimentarios }\end{array}$ & Rango & $\mathbf{N}$ & $\begin{array}{l}\text { Promedio } \\
\text { de puntaje } \\
\text { obtenido } \\
\text { ( } \pm \text { DE) }\end{array}$ & $\%$ & $\mathbf{N}$ & $\begin{array}{l}\text { Promedio } \\
\text { de puntaje } \\
\text { obtenido } \\
\text { ( } \pm \text { DE) }\end{array}$ & $\%$ \\
\hline Optima & $\geq 8$ & 14 & $\begin{array}{c}9,79 \\
( \pm 1,58)\end{array}$ & 33 & 27 & $\begin{array}{c}9,5 \\
( \pm 1,36)\end{array}$ & 64 \\
\hline $\begin{array}{l}\text { Necesidad } \\
\text { de mejorar }\end{array}$ & 4 a 7 & 22 & $\begin{array}{c}5,5 \\
( \pm 1,14)\end{array}$ & 53 & 10 & $\begin{array}{c}5,9 \\
( \pm 1,28)\end{array}$ & 24 \\
\hline No Optima & $\leq 3$ & 6 & $\begin{array}{c}2,33 \\
( \pm 0,82)\end{array}$ & 14 & 5 & $\begin{array}{c}2,8 \\
( \pm 0,44)\end{array}$ & 12 \\
\hline Total & & 42 & & 100 & & & 100 \\
\hline
\end{tabular}

Al comparar las medianas de los puntajes obtenidos antes y después de la intervención en los padres, observamos que existen diferencias significativas entre los grupos. ( $p<0,001$ prueba de Wilcoxon).

\section{DISCUSIÓN}

Al finalizar la intervención, se constató que hubo una disminución en cuanto a niños que tenían obesidad y riesgo de desnutrición, así como un aumento de escolares que contaban con un adecuado estado nutricional, resultados similares se observaron en el estudio de Vázquez $\mathrm{F}$. et. al. en el que escolares entre 8 y 13 años, recibieron una intervención simultánea de ejercicio físico, educación alimentaria y apoyo psicológico, observando una mejoría significativa en el estado nutricional, luego de 3 meses de intervención ${ }^{(16)}$.

Con respecto a las actividades físicas se pudo observar que al final de la intervención, la mayor parte de la población de estudio contaba con buena actividad física, a diferencia del inicio en el cual sólo incluía el 5\%, estos resultados fueron similares a un estudio realizado por Díaz X, en el cual también se produjeron cambios al final de la intervención, ya que inicialmente el mayor porcentaje de la población cursaba con actividad física baja y el menor porcentaje contaba con una actividad física alta, situación que cambió luego de 5 meses de intervención, siendo el 
porcentaje más elevado el que compete a actividad física media y alta, quedando en menos proporción aquellos con baja actividad física ${ }^{(17)}$.

Seguidamente, al analizar si existe asociación entre el estado nutricional de los escolares post intervención y el tipo de actividad física de los mismos tras la intervención, no se encontró relación entre estas variables ( $p=0,465$ Chi cuadrado), resultados similares fueron arrojados por un estudio en el que se analizaron las posibles relaciones entre la Calidad de Vida Relacionada con la Salud (CVRS) con el estado nutricional y los niveles de Actividad Física (AF) y Condición Física (CF) en adolescentes chilenos, de manera independiente e interactiva, el cual mostró que existen relaciones independientes entre los mismos ${ }^{(18)}$.

Por otro lado, para la calidad de hábitos alimentarios de escolares, se halló un interesante cambio luego de la intervención, ya que al comparar las medianas de los puntajes obtenidos antes y después de la intervención en los escolares, se observó que existen diferencias significativas entre los grupos. ( $p<0,001$ prueba de Wilcoxon). Existe otro estudio similar, en el cual Scruzzi G, obtuvo mejores resultados en cuanto a cambios pos-intervención, que podrían deberse al tiempo invertido en el mismo y a una visión integral de la problemática (actividad física, educación alimentaria y apoyo psicológico), con la participación de más actores referentes de la comunidad educativa (docentes, centro de salud y familia). El estudio tuvo una duración inicial de 12 meses, y se emplearon diferentes fases, durante la primera fase se desarrolló un diagnóstico de situación, durante la segunda se realizó la educación alimentarianutricional y en la tercera fase, se estableció un kiosko saludable, de forma a medir también las preferencias alimentarias ${ }^{(19)}$.

Así mismo, con respecto a la calidad de hábitos alimentarios, tanto de los padres como de las madres, al comparar las medianas de los puntajes obtenidos antes y después de la intervención, se observó que existen diferencias significativas entre los grupos ( $p<0,001$ prueba de Wilcoxon), es importante destacar que los hábitos alimentarios de los escolares y madres de los mismos antes de la intervención reflejaron un resultado similar, pero al evaluarlos nuevamente al finalizar las charlas se destacó un mayor cambio en los escolares y menor cambio en las madres, pero aún así significativo en el último grupo. Igualmente, al comparar la calidad tanto de los padres como de las madres no existieron grandes diferencias en cuanto a la alimentación de los mismos. Las referidas diferencias en cuanto a los resultados antes y después de la intervención podrían deberse a que los escolares contaron con 8 charlas informativas, por lo cual tuvieron mayor tiempo de concientización y los padres sólo 2 charlas ${ }^{(20)}$, así mismo, resultados similares fueron arrojados en el estudio de Rodrigo-Cano, en el cual los padres y escolares presentaron cambios significativos en la calidad de hábitos alimentarios, luego de una educación alimentaria(21). No obstante, se podría decir que los niños inclusive llegarían a subir aún más el puntaje de calidad de hábitos, ya que en un estudio se menciona el efecto positivo de las comidas en familia y sobre todo si estos alimentos son elaborados por algún miembro de la familia ${ }^{(22)}$. Por otro lado, siempre teniendo en cuenta el factor tiempo de los padres-niños durante las comidas, en un análisis realizado, igualmente hacen referencia a la importancia de la preparación y realización de comidas en familia, como así también a no hacer otro tipo de actividades durante el acto de comer ya que se puede suponer que no se atiende al tipo ni a la cantidad de comida que se consume ${ }^{(23)}$.

La principal fortaleza de este estudio es el enfoque sobre los cambios en la calidad de hábitos alimentarios ocurridos tras las charlas, ya que hoy en día las intervenciones se realizan a nivel internacional periódicamente, en tanto a nivel país son escasamente realizadas; si bien existen programas y leyes que estipulan el expendio de comidas saludables en el entorno escolar, y teniendo en cuenta que la Escuela Brasil formaba parte del Programa de Alimentación Escolar, que incluye cantina y almuerzo saludable, los niños reflejaron calidad de hábitos alimentarios similares a aquellos que no cuentan con dichos programas y que solo participaron alguna vez de una intervención. Es importante recalcar, que las charlas realizadas fueron semanales y que en dos meses se obtuvieron resultados positivos y cambios significativos. Por 
otro lado, es digno de hacer mención, que los escolares presentaron mucha aceptación en cuanto a la implementación de estas intervenciones, se mostraron muy participativos y activos durante el mismo. Igualmente, los padres en su mayoría estuvieron abiertos y colaborativos a la hora de realizar el test y acompañar a los hijos durante el tiempo de la investigación, sin embargo, una limitante en este aspecto y que se refleja en la calidad de hábitos alimentarios y también en cuanto a la actividad física, sería el factor tiempo, ya que la mayoría contaba con un horario laboral completo. Otra limitante sería que los referidos escolares ya cuentan con programas de alimentación escolar y por ende ya tienen conocimientos básicos sobre alimentación, por lo cual los resultados tal vez serían diferentes en aquellos escolares que no se encuentren dentro de un programa alimentario vigente. I gualmente, es importante destacar que los profesores cumplen también un papel importante dentro del ciclo de intervenciones, generando así marcadas diferencias a la hora del aprendizaje, ya que durante las charlas se observó la diferencia en cuanto a la percepción y participación de los escolares, siendo mayor en una de las secciones, porque aquel curso en el cual el profesor no contaba con dominio del grupo, se hizo más difícil la interacción con los mismos. Finalmente, se puede decir que estos resultados podrían contribuir a futuras investigaciones que pretendan generar cambios en la calidad de hábitos alimentarios como estrategia de prevención de enfermedades a largo plazo, siendo las escuelas el punto focal de intervención por la edad en la que se encuentran los mismos y teniendo en cuenta que están más predispuestos a la obtención de información y a crear hábitos de vida saludable; sería interesante la creación de un proyecto que incluya a las escuelas en la cual se pueda establecer un programa que sea evaluado anualmente, en el que se puedan contemplar charlas semanales de una hora de duración, tratando diferentes temas que tengan que ver con la alimentación y analizar al término del mismo, los cambios generados de manera a seguir fomentando un hábito saludable en la población escolar, que es la más susceptible a los cambios, pudiendo este proyecto crear un antes y un después en el futuro de estos escolares.

\section{AGRADECI MI ENTOS}

A la Lic. Macarena Morínigo, mis agradecimientos, por la predisposición en cuanto a aconsejarme en cada pequeño detalle y transmitir sus diversos conocimientos y experiencias.

A la Lic. Andrea Milena Fernández, Directora de Alimentación Escolar del MEC, por abrirme las puertas y brindarme su valioso tiempo, a los efectos de recabar información para este estudio.

A la Prof. Asunción Vall de Font, Prof. Carolina Rodríguez y Prof. Loli, Docentes de mi querida Escuela Básica $N^{\circ} 3$ República del Brasil, por hacerme sentir como en casa y recibir el apoyo incondicional de la institución en todo momento, este trabajo fue posible gracias a ustedes.

\section{REFERENCI AS BI BLI OGRÁFI CAS}

1. Rivera J, De Cossío T, Pedraza L, Aburto T, Sánchez T, Martorell R. Childhood and adolescentoverweight and obesity in LatinAmerica: a systematicreview. Lancet Diabetes Endocrinol. 2013; 2(4): 321-32.

2. Diez-Canseco F, Saavedra-García L. Programas sociales y reducción de la obesidad en el Perú. RevPeruMedExp Salud Publica. 2017; 34(1): 105-12.

3. Mönckeberg F. Desnutrición infantil y daño del capital humano. RevChilNutr. 2014; 41(2): 173-180.

4. Ortiz-Galeano I, Riquelme M, Chirico C. Sobrepeso, obesidad y factores asociados en pacientes de consultorio de Clínica Médica de un hospital universitario de Paraguay, 2014. Rev. Fac. Cienc. Salud UDES. [Internet] 2016 junio.[acceso 18 de junio de 2020]; 3(2): 102-106. Disponible en: http://dx.doi.org/10.20320/rfcsude s.v3i2.201

5. Ministerio de Salud Pública y Bienestar Social. Plan Nacional de Acción para la Prevención y Control de Enfermedades Crónicas No Transmisibles 20142024[Internet]. Asunción: Dirección de Vigilancia de Enfermedades no Transmisibles; [actualizada en octubre 2014; accesol8 de junio de 2020]. Disponible 
en: http://portal.mspbs.gov.py/dvent/pl an-nacional-de-accion-2014-2024/

6. Ministerio de Salud Pública y Bienestar Social. Guías Alimentarias del Paraguay [Internet]. Asunción: Instituto Nacional de Alimentación y Nutrición; [actualizada en agosto 2015; acceso 18 de junio de 2020]. Disponible en: http://www.inan.gov.py

7. Ministerio de Educación y Ciencias. Programa de Alimentación Escolar del Paraguay - PAEP [Internet]. Asunción: Ministerio de Educación y Ciencias; [actualizada en agosto 2015; acceso 18 de junio de 2020]. Disponible en: https://www.mec.gov.py/cms/?ref= 297017-hacia-una-politica-publica-dealimentacion-escolar

8. Oliva R, Tous M, Gil B, Longo G, Pereira J, García P. Impacto de una intervención educativa breve a escolares sobre nutrición y hábitos saludables impartida por un profesional sanitario. NutrHosp. 2013; 28(5): 15671573.

9. Aparco J, Bautista-Olórtegui W, AsteteRobilliard L, Pillaca J. Evaluación del estado nutricional, patrones de consumo alimentario y de actividad física en escolares del Cercado de Lima. RevPeruMedExp Salud Pública. 2016; 33(4): 633-639.

10. Tarqui-Mamani C, Sánchez-Abanto J, Álvarez-Dongo D, Gómez-Guizado G, Valdivia-Zapana S. Tendencia del sobrepeso, obesidad y exceso de peso en el Perú. Rev. Peru. Epidemiol. [Internet] 2013 diciembre. [acceso 18 de junio de 2020]; 17(3). Disponible en:

https: // www. redalyc. org/articulo. oa? $\mathrm{id}=203$ 129459004

11. Díaz-Beltrán M. Factores influyentes en el comportamiento alimentario infantil. Rev. Fac. Med. [Internet] 2014 mayo. [acceso 18 de junio de 2020]; 62(2): 237-245. Disponible en: http://www.scielo.org.co/pdf/rfmu n/v62n2/v62n2a10.pdf

12. Michels N, Sioen I, Braet C, Eiben G, Hebestreit A, Huybrechts $I$, et al. Stress, emotionaleatingbehaviour and dietarypatterns in children. Appetite. 2012; 59: 762-769.

13. Santomauro $F$, Lorini $C$, Tanini $T$, Indiani L, Lastrucci V, Comodo N,et al. AdherencetoMediterraneandiet in a sample of Tuscanadolescents. Nutrition [Internet] 2014 abril. [acceso 18 de junio de 2020]; 30(11-12): 1379-1383. Disponible

en: http://dx.doi.org/10.1016/j.nut.201 $\underline{4.04 .008}$
14. Godard C, Carlin L, Torres J, Rodríguez M, Leyton B. Salazar G. Nivel de intensidad de las principales actividades físicas de escolares chilenos: determinación por acelerometría. RevChilNutr. 2016:43(3): 247-254.

15. Bergel $M$, Cesani $M$, Cordero $M$, Navazo $B$, Olmedo $S$, Quintero $F$, et al. Valoración nutricional de escolares de tres países iberoamericanos: Análisis comparativo de las referencias propuestas por el International ObesityTaskForce (IOTF) y la Organización Mundial de la Salud. Nutr. clín. diet. hosp. [Internet] 2014 febrero.[acceso 18 de junio de 2020]; 34(1):8-15. Disponible en: https://revista. nutricion.org/PDF/VA LORACION-NUTRICIONAL.pdf

16. Vázquez F, Díaz E, Lera L, Meza J, Salas I, Rojas, et al. Impacto del ejercicio de fuerza muscular en la prevención secundaria de la obesidad infantil; intervención al interior del sistema escolar. NutrHosp. 2018;28(2):347356.

17. Díaz XM, Mena CP, Valdivia-Moral $P$, Rodríguez A, Cachón J. Eficacia de un programa de actividad física y alimentación saludable en escolares chilenos. Hacia promoc. salud. [Internet] 2015 marzo. [acceso 18 de junio de 2020]; 20(1): 83-95. Disponible: http://www.scielo.org.co/pd f/hpsal/v20n1/v20n1a06.pdf

18. Garcia-Rubio J, Olivares P, LopezLegarrea P, Gomez-Campos R, CossioBolaños $M$, Merellano-Navarro $E$. Asociación entre la calidad de vida relacionada con la salud, el estado nutricional (IMC) y los niveles de actividad física y condición física en adolescentes chilenos. NutrHosp. 2015; 32(4) : 1695-1702.

19. Scruzzi G, Cebreiro C, Pou S, Rodríguez Junyent C. Salud escolar: una intervención educativa en nutrición desde un enfoque integral. Cuadernos.info. [Internet] 2014 octubre. [acceso 18 de junio de 2020]; 35: 39-53. Disponible en: https://doi.org/10.7764/cdi.35.644

20. Anaya S, Álvarez M. Factorsassociated withchildren'sfoodpref erences. rev. eleuthera. [I nternet] 2018 enero-junio. [acceso 18 de junio de 2020]; 18(1): 58-73.Disponible en: http://www.scielo.org.co/pdf/eleut/ v18/2011-4532-eleut-18-00058.pdf

21. Rodrigo-Cano S, Soriano J, AldasManzano J. Valoración de la efectividad de la educación alimentaria en niños preescolares, padres y educadores. RevEspNutrHumDiet. [Internet] 2016 
Villagra et al Intervención Educativa-Nutricional sobre hábitos alimentarios aplicada a escolares...

enero. [acceso 18 de junio de 2020]; 20(1): 32 - 39.Disponible en: http://dx.doi.org/10.14306/renhyd. 20.1.182.

22. Turconi G, Moro S, Testa L, Rossi M, Porzio E, Roggi C, et al. What are Children'sBreakfastHabits and HowisBreakfastMealPerceivedbythem? A StudyCarriedout in Pavia, Northernltaly. J NutrHealthSci. [Internet] 2015 mayo. [acceso 18 de junio de 2020]; 2(2): $201 . \quad$ Disponible en: http://www.annexpublishers.co/arti cles/] NH/volume-2-issue-1/What-areChildrens-Breakfast-Habits-and-How-isBreakfast-Meal-Perceived-by-them-A-
Study-Carried-out-in-Pavia-NorthernItaly.pdf

23. Hernández-Rincón E, Severiche-Bueno D, Romero-Mayorga D, López-Sánchez M, Espitia-Franco, Rodríguez-Mancera A.Promoción de alimentación saludable en hogares comunitarios infantiles del municipio de Sopó (Cundinamarca. Colombia) bajo la estrategia de Atención Primaria en Salud. Salud Uninorte. Barranquilla (Col.) [Internet] 2015 septiembre-diciembre. [acceso 18 de junio de 2020]; 31 (3): 514-524. Disponible

en: http://www. scielo.org.co/pdf/sun/v 31n3/v31n3a08.pdf 